\title{
Initiation of JE Vaccination-An Experience: A Mixed Method Study In A Tertiary Care Center of West Bengal, India
}

\author{
${ }^{*}$ Dr Amrita Samanta ${ }^{1}$, DrJadav Chandra Sardar ${ }^{2}$, Dr Prajjwal Sengupta ${ }^{3}$ \\ Dr Anamika Chatterjee ${ }^{4}$, Dr Saibendu Kumar Lahiri ${ }^{5}$ \\ ${ }_{1,2,3,4,5}$ (Dept of Community Medicine, RG KAR medical College and hospital, West Bengal, India) \\ *Corresponding author: *Dr Amrita Samantal
}

\begin{abstract}
Background:JE is an emerging public health problem in India.JE vaccine has been incorporated in the National Immunization Schedule in India following mass vaccination.

Objectives:To explore the experiences gathered during mass vaccination period in a tertiary care center.

Methodology:A mixed method approach consisting of a cross-sectional survey and qualitative study was adopted. The qualitative study was included to obtain more in-depth understanding of the experiences and perspectives of frontline workers and the team leader in form of in depth interview of LMO of satellite center and FGDamong all PHNS involved in the activity.

Result:Among 2437 vaccinees surveyed, majority belonged to 1-5 years age group followed by 6-10 years. Only half of the caregivers of surveyed population knew the purpose of vaccination and 6\%perceived that the vaccine prevents 'Dengue'. No AEFI was reported during and one months following the mass vaccination.Qualitative approach revealed huge enthusiasm among the people about the vaccine, lack of staff and space to cater large age group section and inappropriate rush during the vaccination sessions and impairment of routine vaccination during the mass vaccination period.

Conclusion:Thoroughly planned systematic IEC activities and prior deployment and orientation of staffs are pre requisites for conducting suchlarge scale mass vaccination programme.
\end{abstract}

Key words: FGD, In depth interview, JE vaccine, Mass vaccination,

\section{Introduction}

$\mathrm{JE}$ is an emerging public health problem with complex eco-epidemiological characteristics that poses a serious challenge in terms of prevention and control. A leading viral cause of Acute Encephalitis Syndrome (AES) is highly prevalent in India.[1]In 2014,1661 cases of JE were reported from 15 states and union territories of India, out of which 293 (17.6\%) died. Assam, West Bengal, Uttar Pradesh (UP) and Jharkhand reported maximum number of cases. [2]Japanese Encephalitis (JE) is a mosquito borne zoonotic viral disease. The virus is maintained in natural hosts such as animals, birds, pigs and Ardeid birds. Pigs \& wild birds are reservoirs of infection and are called as amplifier hosts in the transmission cycle, while man and horse are dead end hosts. The virus does not cause any disease among its natural hosts and transmission continues through vishnui group of culex mosquitoes. The incubation period ranges from 5 to 14 days. The disease affects the Central Nervous System and can result in severe complications, seizures and even death. The Case Fatality Rate (CFR) of this disease is very high and survivors may suffer from various degrees of neurological sequeale. An estimated $25 \%$ of the affected children die, and among those who survive, about 30-40\% suffers from physical \& mental impairment. The children suffer the highest attack rate because of lack of cumulative immunity due to natural infections.[1]

The first evidence of presence of JE virus dates back to 1952 in the Nagpur subdivision of Maharashtra. In 1955, JE was clinically diagnosed for the first time in Vellore, Tamil Nadu. In subsequent years, outbreaks were reported in various States and UTs in the country. The first major JE epidemic was reported from the Burdwan and Bankura districts of West Bengal in 1973 followed by another outbreak in 1976. Outbreaks have been reported from many states in following years.[3]

The most promising preventive tool is JE vaccine, which, though available since 1941, remained out of reach for most countries due to small production capacity and relatively high cost. Fortunately, the development and increasing availability of new JE vaccines in recent yearshave made the control of JE more of a reality. [4]

Based on the recommendations of the Bi-Regional Consultation on Japanese Encephalitis (WHO SEA/WPR and PATH, Thailand, March-April 2005), Government of India has decided on the following strategy for the introduction of the JE vaccine in the endemic districts in India:

- A one-time mass campaign targeting all children in the age group of 1-15 years in the districts. 
- Followed by integration of the JE vaccine into the Routine Immunization Program to cover the new cohort (children attaining more than 1 year of age) in the districts covered previously under the JE vaccination campaign. These children would be administered JE vaccine between 1-2 years of age along with the DPT booster dose, under the Routine Immunization Programme.

- A special campaign has been planned for 2010 in selected districts in the country to cover left outs and new cohorts.

- $\quad$ Age distribution pattern of the lab confirmed JE cases will be reviewed to further inform strategy.

JE vaccination in India started in 2006 following large outbreaks of JE in some districts of Eastern UP and Bihar. Children between the age group of 1 to 15 years were vaccinated with a single dose of Chinese live attenuated SA-14-14-2 JE vaccine [3].In 2011, the same SA-14-14-2 JE vaccine was introduced in the routine immunization under Universal Immunization Program (UIP) in the 181 endemic districts as a single dose at 16 to 18 months at the time of 1 st booster of DTP vaccine. In 2013, another dose of SA-14-14-2 vaccine was added at 9 months of age along with measles vaccine [4]. However, except for a few reports, no research material sharing experiences or SWOT analysis of introduction and implementation of the newer vaccine in India was available.Keeping the above in mind, the current study was undertaken during the mass campaign phase of JE vaccination for 1- 15 year age group in Kolkata district of West Bengal duringthe last week of December,20161st week of March, 2016 with the aim of understanding the experiences in the initiation phase in a tertiary care center of West Bengal.

\section{Materials and Methods}

2.1 Study setting: The study was conducted in immunization clinic and Urban Health Training Center (UHTC) of RG Kar Medical College \& Hospital, Kolkata. The UHTC is a satellite center of RG Kar Medical College situated in a Bag-bazar slum which is the urban field practice area of Department of Community Medicine.The immunization clinic is situated in the OPD complex and is staffed by four Public Health Nurses (PHNs) and one Group D staff. Routine vaccination is carried out 6 days per week in immunization clinic. In UHTC, routine vaccination is performed every Wednesday and it has one Lady Medical Officer (LMO) in charge,two PHNs and one Group Dstaff. Interns and Post Graduate Trainees(PGTs) are posted at both the centers. During the mass campaign phase, JE vaccination was conductedat all working days in the immunization clinic and in four working days per week in UHTC excluding OPD days.After the mass campaign, JEvaccine was introduced into the routine immunization schedule in subsequent months as per Government policy in both the centers.

2.2 Type of Study:A mixed methods approach was adopted for the current study, consisting of a crosssectional survey and qualitative study. The qualitative study techniqueswere included to obtain a more in-depth understanding of the experiences and perspectives of key person and HEWs engaged for the immunization activity. In depth interview of LMO was conducted and Focus Group Discussion (FGD) was done involving all six PHNs working in immunization clinic and UHTC.

2.3 Period of study:In the cross sectional survey data was collected using a predesigned, pretestedand semi structured questionnaire by interns and postgraduate trainees posted in immunization clinic and UHTC during the JE mass vaccination campaign from last week of December 2015 to First week of March 2016. The qualitative study was done in the month of April, 2016 after completion of mass vaccination program.

\subsubsection{Cross Sectional Survey}

\section{Methods of Data Collection}

The questionnaire comprised of socio demographic characteristics (age, gender, educational status, status of parents,occupation of parents, monthly family income, type of family,residence andsocio economic status as permodified B G Prasad scale), routine immunization status and knowledge about the disease for which vaccine was given(Why is the vaccine being given?) Vaccinees were observedforhalf an hour following vaccination in the clinic for any immediate Adverse Events Following Immunization (AEFI).

The questionnaire was pretested among 20 parents of eligible vaccinees not included in the final survey and was modified accordingly.

\subsubsection{Qualitative Study}

The qualitative investigation comprised one in-depth interview of the lady medical officer who was the key person and one FGD in Bengali involving all public health nurses (6 persons). The interview and FGD were conducted in workplaces of the staff.During data collection, field notes were taken, interviews were recorded by Audio Digital Recorder (ADR) and non-verbal and gestural cues were observed.Aninterview guide was prepared for in-depth interviews. The in-depth interview was expanded upon the quantitative survey and specifically explored the following areas: the overall experience of mass vaccination program, difficulties 
faced, any AEFI reported, strength and gap of the activities, and suggestions and opinion for improvement of the program.

The FGD lasted for one and half hours and based on the similar themes of in depth interview.

\subsection{Study population:}

Every second person registered for JE vaccination during this campaign session was targetedand sought informed verbal consent from accompanying caregivers or parents. A Total of 5057 persons were vaccinated in the mass campaign and 2530 persons were approached by study team of which 2437 accompanied parents or care givers gave consent to interview.

Inclusion criteria for cross sectional survey:

1. Children aged $1-15$ years

2. Those whose parents or caregivers provided informed verbal consent

Inclusion criteria Qualitative Study:

Lady medical officer in charge of UHTC and all PHNs engaged in immunization activity were purposively chosen for qualitative assessment.

3.6 Ethical consideration:Informed verbal consent was obtained from all the respondents before the interview maintaining necessary confidentiality.

\section{Data analysis}

4.1Cross sectionalsurvey: The collected data in cross survey was analyzed using SPSS (Version 20.0, Chicago, IL) and expressed in proportion.

4.2 Qualitative Study: All the collected data were translated into English and transcribed verbatim by the researcher and research assistant independently. After the verbatim transcription and translation, the data were compiled, read and re-read of the transcripts and emerging ideas were listed. Then codes and categories for the listed ideas were created. Recoding was done when necessary. Themes were generated from these categories. The transcripts were also given to anindependent public health specialist, who was also an expert in qualitative research methodology to independently create codes and categories, and identify emerging themes. A consensus meeting between the researcher and the public health specialist was held, where the categories and themes identified were compared, revised and then used as research findings for this study.

\section{Results}

It was found that most of the study population belonged to $1-5$ year age group (53.2\%), followed by 6 10 years $(38.8 \%)$ and $11-15$ years age group. Gender difference among the study population is not appreciable (male-51.2\% female-48.8\%).Parents of most of the study population were middle level passed (mothers-26\%, father-25.6\%).Almost all (99\%) study population were from urban area.Among the fathers, most of them are unskilled workers (32\%) followed by self-employed or business (27\%). Among the mothers most of the mothers were home makers(71.8\%).Most of the study population belonged to lower middle class(34.5\%) followed by middle class (28.2\%) as per Modified B G Prasad's scale.[5](Table-1)

Among the study population, caregivers of $96.1 \%$ vaccinee reported past history of routine immunization and 96.6\% had BCG scar.(Table-2)

Only half of the caregivers of the vaccinees knew the name of the disease prevented by the vaccine. (Fig1)Nearly 6\% reported that JE vaccine prevents Dengue.Regarding source of information, highest number of study population got information of the mass vaccination from UHTC (40.9\%) followed by neighbors (15.3\%).(Table-3)No adverse event following immunization except mild pain during injection was reported within half hour of vaccination. This was further substantiated by qualitative study. No vaccinee reported any adverse event within one months of vaccination.

From FGD and in-depth interview following themes wereidentified and findings were grouped into the following emerged themes

1. Overall experience of the staffs regarding mass vaccination

2. Difficulties faced

3. Suggestion of the staff for further improvement in future.

Overall experience of the staffs regarding mass vaccination:The in depth interview revealed that the overall enthusiasm was appreciable among beneficiaries regarding JE vaccination. People came not only from the catchment area of the UHTC but from areas outside Kolkata. The outsiders were informed by theirrelatives 
who were residents of Bag bazar slum. Experience from R G KMC revealed that people have more faith on Tertiary care center than short term camps in the locality especially for some injectable vaccines. School going children came being absent to their respective schools on weekdays.

\section{Difficulties faced:}

1. Statement of one of the PHNswas 'there was massive mismanagement in the earlyperiod of the vaccination' No extra staffs were deployed for the peak rush hours.Long queues and less space for sitting arrangement of vaccine resulted in dissatisfaction, intolerance and aggression among the vaccinees and their caregivers. The broad age group (1-15 years) of vaccinees that resulted in such huge footfalls was another reason behind mismanagement.

2. Routine immunization activities were jeopardized temporarily.

3.Many people had to be returned back due short supply of vaccines compared to huge clients in first 10- 15 days where as vaccine supply was surplus in later part of the period. The PHNs stated that "we were not sure how much vaccine to be indented from store as number of vaccinee could not be predicted from beforehand."

4.Due to rush they were unable to impart the required health education messages on the JE vaccine, AEFI, routine vaccination to the population as stated by one of the PHN 'due to long queue and rush we could not tell to all vaccinee or their parentsor caregivers regarding the vaccine, its AEFI etc.'

\section{Suggestions}

From the FGD and In-depth interview following suggestions for future endeavor came up.

1.Proper intensified systematic IEC campaign before any such mass vaccination should be done so that people will be aware that every eligible person will get the service, reason behind the service and the particular center for getting the service .School authority might be chosen as IEC partners for spreading awareness for school going beneficiaries .

2. Extra trained staffs must be deployed for this extra load of activities and steady routine services. Prior orientation of the staffs on-duty is also a prerequisite.

3. Planning of immunization session could be more pragmatic. The LMO opined that 'it will be helpful if the age-group of 1-15 years was broken into smaller groups and fixed dates for each age group is designated beforehand.'

4. One of the PHN told that 'arrangements for school going beneficiaries could be made on Saturdays or Sundays which could reduce school absenteeism'

\section{Discussion:}

So far, 155 out of 181 identified JE endemic districts have been covered under JE campaign and overall 10.8 crore children have been immunized with JE vaccine through campaigns [3].

Meagre research is available on JE mass vaccination. The present study revealed that the mass campaign covered mostly lower to middle class of population.Strategy to cover upper economic group should be there in the vaccination policy.

In current study, it was observed that about $50 \%$ study population were aware about the disease against which the vaccine was provided. The coverage evaluation survey in Karnataka district also revealed low level $(42 \%)$ of awareness.[6]Another point of concern is that $6 \%$ of population thought that the vaccine prevents dengue. The contents of IEC material must focus on the Japanese encephalitis, the disease and its signs and symptoms, as misconception might hinder the successful routine vaccination against JE .Especially, since dengue has currently reached an epidemic proportion in Kolkata, false sense of security with JE vaccination may complicate the problem further.The Karnataka study[6] also reported $4 \%$ AEFI with the same vaccine though no immediate AEFI was observed in the current study and delayed AEFI was reported within one month of vaccination. However the coverage evaluation survey was community based contrary to the current clinic based study.It hasalso been found that most of the vaccineesobtained information from the staffs of the Urban Training Center followed by mass media. So it is imperative that the staffs are working very efficiently regarding masscatch- up campaign.

The qualitative aspect of this study also showed that though people had obtained the information of a Government sponsored new vaccine, most of them were unware about the purpose of vaccination and disease prevented by the vaccine. As quoted by the LMO, 'Somehow they had the information that some vaccine was being offered by the Government to 1-15 years children free of cost from this center. Most of them were unaware about the reason and disease for which the vaccine was being given. Most of them were in a hurry because they thought the vaccine would not be available for all if they were late' 
The qualitative part of studyalso threw some light on the probable factors behind the inordinate rush in first few days of vaccination such as lack of information about the duration of mass vaccination period,fear of scarcity of vaccine stock, aggregation of beneficiaries from areas other than catchment area in spite of presence of temporary centers in their area and large age group of beneficiaries. In spite of previous and concomitant JE mass vaccination in their locality, people were rushing to the UHTC probably owing to their long-lasting faith on UHTC and RG Kar medical college. This huge rush caused extra burden to the existing health system which could be tackled by prior systematic IEC campaigning and planning of sessions.

\section{Conclusion}

The study had many limitations.It was institutional based and cross sectional. Had it been a community based and longitudinal one, AEFI and community awareness could be ascertained in depth. Further in depth longitudinal research work involving wider population group is required to assess the awareness of community,coverage, effectiveness and AEFI associated with JE vaccineespecially in West Bengal which a major endemic state .The strength of the study was that it covered a large number of target population in limited period of time and the qualitative aspects of the study was able to find out the view points of the health workers directly involved in the service. The study revealed that prior systematic planned IEC programme, orientation of staffs, deployment of staffs and necessary logistics are required for any such future activity.

\section{Acknowledgement}

The authors express the gratitude to all the study participants, the PHNs and Dr DipikaHalder, Lady Medical Officer of UHTC, for their whole-hearted co-operation.We are also thankful to all internees and post graduate students of Dept of Community Medicine for extending their help in data collection.

Table 1. Sociodemographic characteristics of vaccinees $(\mathrm{N}=2437)$

\begin{tabular}{|c|c|}
\hline Variables & Number $(\%)$ \\
\hline \multicolumn{2}{|l|}{ Age Group (Years) } \\
\hline $1-5$ & $1298(53.2)$ \\
\hline $6-10$ & $750(30.8)$ \\
\hline $11-15$ & $389(16.0)$ \\
\hline \multicolumn{2}{|l|}{ Gender } \\
\hline Male & $1247(51.2)$ \\
\hline - $\quad$ Female & $1190(48.8)$ \\
\hline \multicolumn{2}{|l|}{ Residence } \\
\hline - $\quad$ Urban & $2413(99.0)$ \\
\hline • $\quad$ Rural & $24(1.0)$ \\
\hline \multicolumn{2}{|l|}{ Educational Status of Father } \\
\hline $\begin{array}{ll} & \text { Illiterate } \\
\end{array}$ & $205(8.4)$ \\
\hline Just literate & $46(1.9)$ \\
\hline Primary & $431(17.6)$ \\
\hline Middle Class & $623(25.6)$ \\
\hline Secondary & $577(23.7)$ \\
\hline Higher Secondary & $546(22.4)$ \\
\hline - $\quad$ Graduate \& Above & $9(0.4)$ \\
\hline \multicolumn{2}{|l|}{ Educational Status of Mother } \\
\hline • $\quad$ Illiterate & $165(6.8)$ \\
\hline Just literate & $195(8.0)$ \\
\hline Primary & $455(18.7)$ \\
\hline Middle Class & $633(26.0)$ \\
\hline Secondary & $479(19.7)$ \\
\hline Higher Secondary & $502(20.6)$ \\
\hline - $\quad$ Graduate \& Above & $8(0.2)$ \\
\hline \multicolumn{2}{|l|}{ Occupation of Father } \\
\hline • $\quad$ Unskilled Worker & $761(31.2)$ \\
\hline Self Employed or business & $657(27.0)$ \\
\hline Service & $630(25.9)$ \\
\hline Skilled Worker & $305(12.5)$ \\
\hline Professional & $18(0.7)$ \\
\hline Unemployed & $5(0.2)$ \\
\hline Not revealed & $61(2.5)$ \\
\hline \multicolumn{2}{|l|}{ Occupation of Mother } \\
\hline - $\quad$ Homemaker & $1810(74.3)$ \\
\hline Unskilled Worker & $518(21.3)$ \\
\hline
\end{tabular}


Initiation of JE Vaccination-An Experience: A Mixed Method Study In A ....

\begin{tabular}{|ll|l|}
\hline$\bullet$ & Skilled Worker & $28(1.1)$ \\
\hline$\bullet$ & Self Employed & $39(1.6)$ \\
\hline$\bullet$ & Service & $23(0.9)$ \\
\hline$\bullet$ & Professional & $19(0.8)$ \\
\hline Socioeconomic Status* & \\
\hline$\bullet$ & Lower & $309(12.6)$ \\
\hline$\bullet$ & Lower Middle & $838(34.5)$ \\
\hline$\bullet$ & Middle & $688(28.2)$ \\
\hline$\bullet$ & Upper Middle & $384(15.8)$ \\
\hline$\bullet$ & Upper & $157(6.4)$ \\
\hline$\bullet$ & No Response & $61(2.5)$ \\
\hline
\end{tabular}

*Modified BG Prasad's Scale,2016

Table 2.Routine Immunization status of study population after birth $(\mathrm{N}=2437)$

\begin{tabular}{|c|c|}
\hline Past History of Routine Immunization & Number $(\%)$ \\
\hline - $\quad$ Present & $2342(96.1)$ \\
\hline Absent & $4(0.1)$ \\
\hline Do Not Know & $91(3.8)$ \\
\hline BCG Scar & Number $(\%)$ \\
\hline - $\quad$ Present & $2355(96.6)$ \\
\hline Absent & $82(3.4)$ \\
\hline Availability of Card & Number $(\%)$ \\
\hline - $\quad$ Yes & $2383(97.8)$ \\
\hline$\bullet$ & $54(2.2)$ \\
\hline
\end{tabular}

Table 3.Distribution of study population according to source of knowledge about the vaccine. $(n=2437)$

\begin{tabular}{|c|c|c|}
\hline & Source of Knowledge & Number $(\%)$ \\
\hline$\bullet$ & Urban Training Health Centre & $996(40.9)$ \\
\hline$\bullet$ & Neighbours & $373(15.3)$ \\
\hline$\bullet$ & Mass media & $215(8.8)$ \\
\hline$\bullet$ & Poster & $152(6.2)$ \\
\hline$\bullet$ & Doctor & $126(5.2)$ \\
\hline$\bullet$ & Mass media & $215(8.8)$ \\
\hline$\bullet$ & School & $87(3.7)$ \\
\hline$\bullet$ & Local People & $87(3.7)$ \\
\hline$\bullet$ & Family Member & $53(2.2)$ \\
\hline$\bullet$ & Peer Group & $42(1.7)$ \\
\hline$\bullet$ & Health Worker & $30(1.2)$ \\
\hline$\bullet$ & Nursing Staff & $10(0.4)$ \\
\hline$\bullet$ & Local Club & $9(0.4)$ \\
\hline$\bullet$ & Political Party & $3(0.1)$ \\
\hline
\end{tabular}




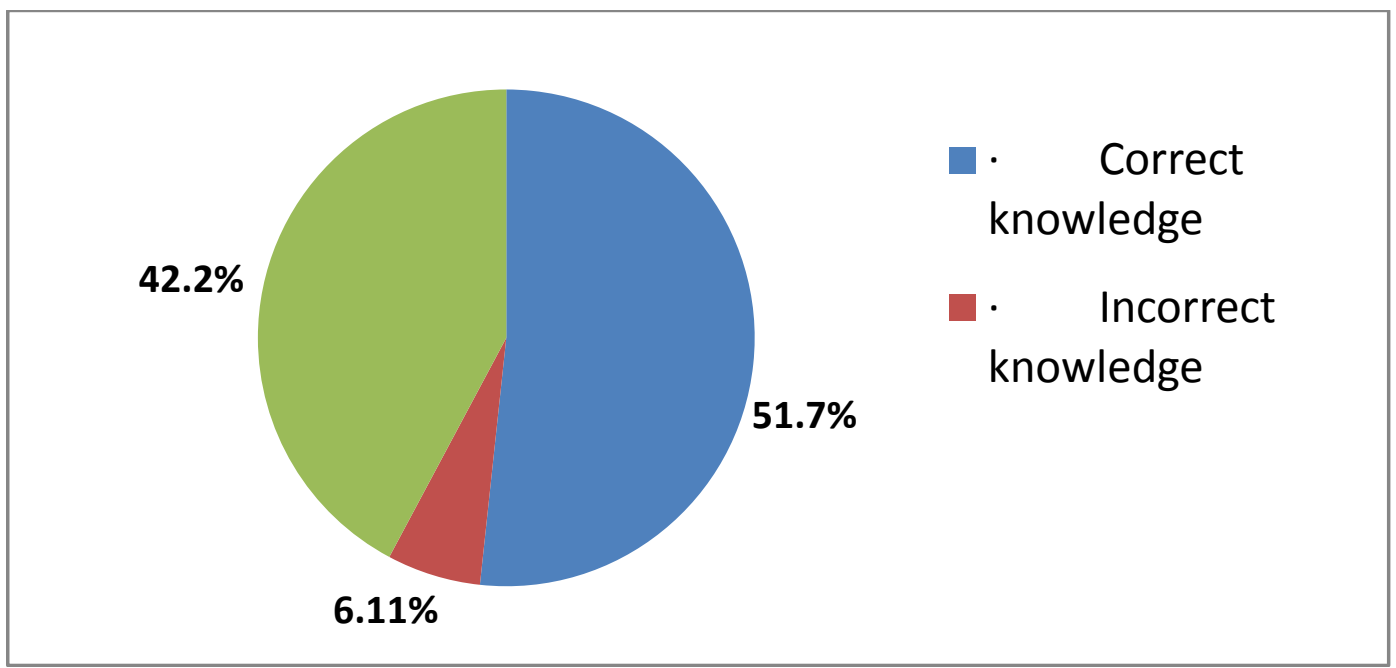

Fig-1, Knowledge of study population about the name of the disease that will be prevented by the vaccine $(n=2437)$

\section{References}

[1]. Directorate of national vector borne disease control program. Details of AES/JE cases and Ddeaths from20082014.New Delhi: Ministry of Health \& Family Welfare, Government of India.Availablefrom:http://nvbdcp.gov.in/Doc/je-aes-cd-May15.pdf. Last accessed : 12 February,2017.

[2]. Operationalguidelines for national programme for prevention and control of Japanese Encephalitis/ Acute Encephalitis Syndrome. New Delhi: Ministry of Health \& Family Welfare, Government of India 2014.

[3]. Operational guide for Japanese encephalitis vaccination in India..New Delhi: MoHFW, Government of India 2010.

[4]. Universal Immunization Program, Immunization Division .New Delhi: MoHFW, Government of India. Available rom:http://www.nhp.gov.in/sites/default/files/pdf/immunization_uip.pdf..Last accessed 25 February 2017

[5]. Shaikh Z, Pathak R.RevisedKuppuswamy and B G Prasad socio-economic scales for 2016. Int J Community Med Public Health 2017;4:997-9

[6]. K Ravi Kuma, RiyazBasha, B R Harish, TV Sanjay, MVinay,Prabhu Suresh et all. A coverage evaluation survey of je vaccination in two districts of Karnataka, J. Commun. Dis, 42(3), 2010 ,179-184

* Dr. Amrita Samanta. "Initiation of JE Vaccination-An Experience: A Mixed Method Study In A Tertiary Care Center of West Bengal, India." IOSR Journal of Dental and Medical Sciences (IOSR-JDMS) 16.7 (2017): 20-26. 